\title{
Breast cancer prevention in older women: an algorithm to choose an optimal preventive agent
}

\section{Burcu Z Ozdemir \\ Joel B Goodin \\ Donald L Bodenner}

Department of Geriatrics, University of Arkansas for Medical Sciences, Little Rock, AR, USA
This article was published in the following Dove Press journal: Breast Cancer - Targets and Therapy

Background: Breast cancer is a significant cause of morbidity and mortality in older women. The current study presents new, comprehensive guidelines for providing chemoprevention to older women.

Objective: The objective of this study was to develop and pilot test a chemopreventive choice algorithm to assess its feasibility for older women at high risk of breast cancer.

Design: The study observed outcomes of 23 older adult females being treated with one of the four different chemopreventive agents. A novel algorithm protocol was utilized for individualized chemopreventive selection.

Setting: The study was conducted in a high-risk outpatient clinic for older women.

Participants: Older outpatient females at high risk $(\mathrm{N}=23)$ were offered chemopreventive options based on individual criteria.

Intervention: Literature review for breast cancer chemopreventive agents informed our development of a logic-based algorithm to guide treatment protocol and chemopreventive choice optimization. Selective estrogen receptive modulators (SERMs) were avoided in women with endometrial cancer risk (ie, pre-hysterectomy individuals), but used in women with low thromboembolic event (TE) risk. Raloxifene was used with osteoporotic women. Aromatase inhibitors (AIs) were used in women with high TE risk. Women without TE risks are advised to take SERMs. When bone density decreased due to AI use, women were switched to raloxifene. Measurements/results: Of 23 participants of age ranging from 59 to 80 years (mean=72.6), two women developed estrogen receptor-positive breast cancer. Two participants, one who declined chemoprevention and one treated with an AI, developed breast cancer. All initial chemopreventive agents were selected according to the algorithm. Although minor adverse events occurred, each was managed by discontinuation or replacement of the chemopreventive agent. Discontinuation was most commonly due to side effect concerns or cost rather than experienced side effects.

Conclusion: Outcomes of the initial utilization of the chemopreventive agent choice algorithm support the viability of the protocol, but further evaluation with a larger and more diverse sample is required.

Keywords: aging, breast cancer, prevention, chemoprevention

\section{Introduction}

Breast cancer is a significant cause of morbidity and mortality in older women. ${ }^{1}$ Females $>60$ years are twice as likely to die from breast cancer compared to younger women. ${ }^{2}$ In the USA, breast cancer mortality has steadily decreased since $1975 ;{ }^{3}$ however, mortality risk remains high $(\mathrm{N}=40,000 ; 14 \%),{ }^{4}$ especially in older women. ${ }^{2}$ Current treatment options are unable to completely address the increased risk of breast cancer mortality
Correspondence: Burcu Z Ozdemir Department of Geriatrics, University of Arkansas for Medical Sciences, 6 Latour Lane, Little Rock, AR 72223, USA

$\mathrm{Tel}+\mathrm{I} 50$ I 23I 979

Email ozdemirburcu@uams.edu 
among older women resulting in a need for primary prevention alternatives. It is possible that some of the breast cancer occurrence can be prevented in high-risk women. ${ }^{5}$ Evidencebased clinical tools such as Gail risk index (GRI) allow for the calculation of breast cancer risk to identify high-risk women. ${ }^{6}$ Raloxifene, tamoxifen, exemestane, and anastrozole are chemopreventive options all of which have been identified for this indication with dramatic breast cancer risk reduction. ${ }^{5,7-9}$ Older women are particularly good candidates for prevention as they have a higher risk for breast cancer and are beyond their reproductive years. Nevertheless, pharmaceutical side effects could complicate chemoprevention implementation. Tamoxifen has demonstrated increased endometrial cancer risk and increased the thromboembolic risk for women $>50$ years of age. ${ }^{5}$ Aromatase inhibitors (AIs), such as anastrozole and exemestane, showed no increased risk for endometrial cancer, thromboembolic events (TEs), or fracture; however, both the agents have been associated with decreased bone density over long-term use. ${ }^{8,9}$ The purpose of the current study was to develop a predictive model to identify the appropriate chemopreventive agent to help minimize the incidence of side effects. According to the Food and Drug Administration criteria, 7.4 million women aged 60-79 years were eligible, but untreated, for breast cancer prevention. ${ }^{10}$ Primary care physicians rarely assess breast cancer risk and prescribe chemoprevention drugs for risk reduction. ${ }^{11-13} \mathrm{~A}$ recent meta-analysis suggested that concerns about adverse effects were correlated with underuse of breast cancer chemoprevention. ${ }^{11}$ Reducing medication side effects could increase the preventive medication acceptance by patients and physicians. Choosing the optimal preventive agent with the least possible side effects for a particular patient through careful clinical evaluation can be made possible with an algorithm. Our objective was to develop a clinical algorithm to choose an optimal preventive agent to minimize potential side effects and pilot test the feasibility of this algorithm.

\section{Methods}

The primary investigator (PI) established a specialized highrisk clinic for older women, in a tertiary academic geriatrics outpatient clinic wherein she served as the sole dedicated provider. During that time, she developed an algorithm for optimal selection of chemoprevention treatment using evidence-based literature review. We present the algorithm as a viable tool to enhance breast cancer prevention.

All patients received standard treatments based on their clinical characteristics and as recommended by the United States Preventive Task Force. No experimental treatment or methods were used in this observational study; therefore, institutional review board approval was not required.

\section{Subjects}

A referred older female sample of high-risk outpatients for breast cancer having GRI of $\geq 1.6 \%$ for 5 -year risk ( $\mathrm{N}=23$ ) was offered chemopreventive options based on each individual's criteria. One $(\mathrm{N}=1)$ individual declined. For over 3 years (April 2013 to October 2016) at 0.5 days per month, the PI provided a review of medications, chronic medical problems, past surgeries, family history, breast examinations, risk assessment, and counseling. Data collected from electronic medical records included patients' age, medical problems, current medications, hysterectomy status, history of osteoporosis, and GRI, ${ }^{6}$ which uses online survey responses to calculate breast cancer risk. A 5 -year risk of $\geq 1.6 \%$ is considered to be the minimum threshold for the use of preventative medications. ${ }^{14}$ Patients in the high-risk clinic were offered chemoprevention in accordance with United States Preventative Services Task Force recommendations that physicians engage in shared, informed chemopreventive decision-making with women who are at increased risk for breast cancer. ${ }^{14}$

\section{Literature search}

A computerized literature search was conducted using PubMed to assess the current status of breast prevention strategies and prevention guidelines. The search covered peerreviewed articles limited to clinical trials and meta-analyses published between January 1990 and December 2017. The search terms used were breast cancer prevention and breast cancer prevention algorithm.

\section{Algorithm design}

The PI developed a logic-based algorithm describing a decision-making process based on associated screenings (Figure 1). The algorithm accounts for the evidence of most concerning and common side effects of a particular agent according to each patient's history and medical conditions.

\section{Step I}

Referred women are evaluated for future breast cancer risk using GRI. ${ }^{6}$ Five-year risk over $1.6 \%$ is considered the cutoff for chemoprevention discussion according to USPTF recommendations. ${ }^{14}$

\section{Step 2}

Freedman et $\mathrm{al}^{15}$ described that hysterectomy status strongly affects benefit/risk index for SERMs treatment. Tamoxifen 
Algorithm for determining individuated preventive medication choice: a novel approach to screening females aged 75 and older

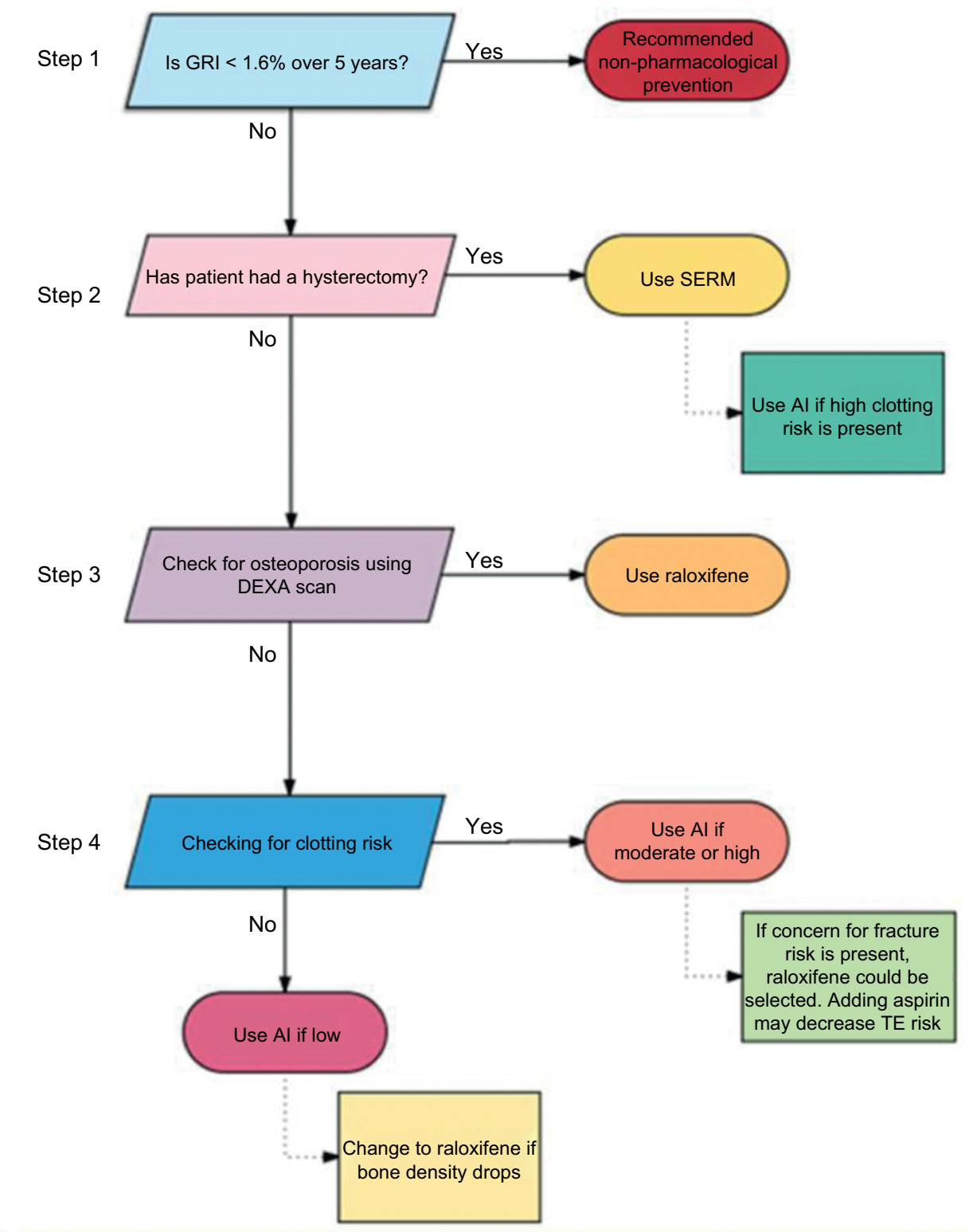

Clinician-patient discussion (prior to chemoprevention initiation)

1. Advise regarding lifestyle modifications such as exercise, avoiding alcohol use and hormone replacement therapy, and maintaining a healthy weight.

2. Discuss side effects and risk of medication versus risk of breast cancer.

Figure I Chemopreventive choice optimization algorithm.

Note: Solid lines indicate strong evidence is present. Dotted lines indicate weaker evidence is present for this recommendation.

Abbreviations: Al, aromatase inhibitor; DEXA, dual-energy x-ray absorptionmetry; GRI, Gail risk index; SERM, selective estrogen receptive modulator; TE, thromboembolic event.

may increase endometrial cancer risk for women aged over 50 years. ${ }^{15}$ However, the same study showed that raloxifene has better benefit/risk index for women aged over 50 years with an intact uterus. For this reason, our algorithm does not recommend tamoxifen for women who have not had a hysterectomy.

\section{Step 3}

Since raloxifene has a better benefit/risk index, and it is an approved treatment to improve bone mineral density, it is recommended for women with osteopenia or osteoporosis regardless of hysterectomy status. 


\section{Step 4}

SERMs such as tamoxifen and raloxifene may increase TEs. ${ }^{16}$ SERMs are reserved for women who are at low-risk for TEs. AIs do not increase thromboembolic risk; hence, women who are at high risk for thromboembolic or cardiovascular events are advised to take AIs. ${ }^{89}$ Thromboembolic risk factors are assessed using the patient's Caprini risk score. ${ }^{17}$

\section{Side shoot at step 4}

In one patient, exemestane had to be stopped due to declining bone density while on this treatment. This patient was switched to raloxifene with the addition of aspirin to her regimen to address both increased cardiovascular risk and decreasing bone density. Barrett-Connor et al showed that raloxifene did not significantly affect the risk of coronary heart disease but increased risk of venous thromboembolism and fatal stroke. ${ }^{18}$

\section{Results}

\section{Literature search results}

The literature search identified 2,464 publications for breast cancer prevention and 341 publications for breast cancer prevention algorithm. This literature was evaluated for relevance. The most relevant articles were reviewed and served as the basis of this algorithm. As the algorithm was applied to subjects, changes were made according to real-life challenges during the study. The algorithm was finalized at the end of the study.

\section{Subjects}

Female participants $(\mathrm{N}=23)$ of age ranging from 59 to 80 years $($ mean $=72.6)$ were $60.9 \%(\mathrm{~N}=14)$ post-hysterectomy and averaged 11.9 medications and 6.3 medical problems. While 5-year GRIs ranged from $1.7 \%$ to $17.0 \%$ (median=4.2\%), lifetime GRIs ranged from $2.1 \%$ to $49.0 \%$ (median=11.5\%). Duration of follow-ups ranged from 1 to 42 months (median=17.6 months), although initial follow-up occurred within the first year after the visit to the high-risk clinic. Chemotherapeutic choice treatment processes are shown in Figure 2. All minor adverse events $(\mathrm{N}=6$; Figure 3) were managed by discontinuation or replacement of the chemopreventive agent. Discontinuation was most commonly due to side effect concerns or cost (eg, raloxifene).

\section{Clinical characteristics and follow-up}

Table 1 summarizes the clinical characteristics of the patients. During follow-up, six patients stopped raloxifene. Four of the six patients stopped raloxifene due to the cost of this medication. One patient stopped it due to light vaginal bleeding. Another patient stopped it as she has started to worry about side effects without actually experiencing any. Two patients stopped exemestane, one due to increased arthritic pain and the other due to declining bone density during follow-up. Two patients stopped tamoxifen, one due to hair loss and the other due to elevated liver function tests.

Although all the 23 patients were appropriate candidates for chemoprevention, one patient with a past history of breast cancer declined chemopreventive treatment and was found to have developed contralateral breast cancer upon follow-up. A second woman developed breast cancer 3 months after initiating an AI, identified as stage 0 ductal carcinoma in situ and was treated with lumpectomy alone. The tumor was possibly present, but undetected, before the
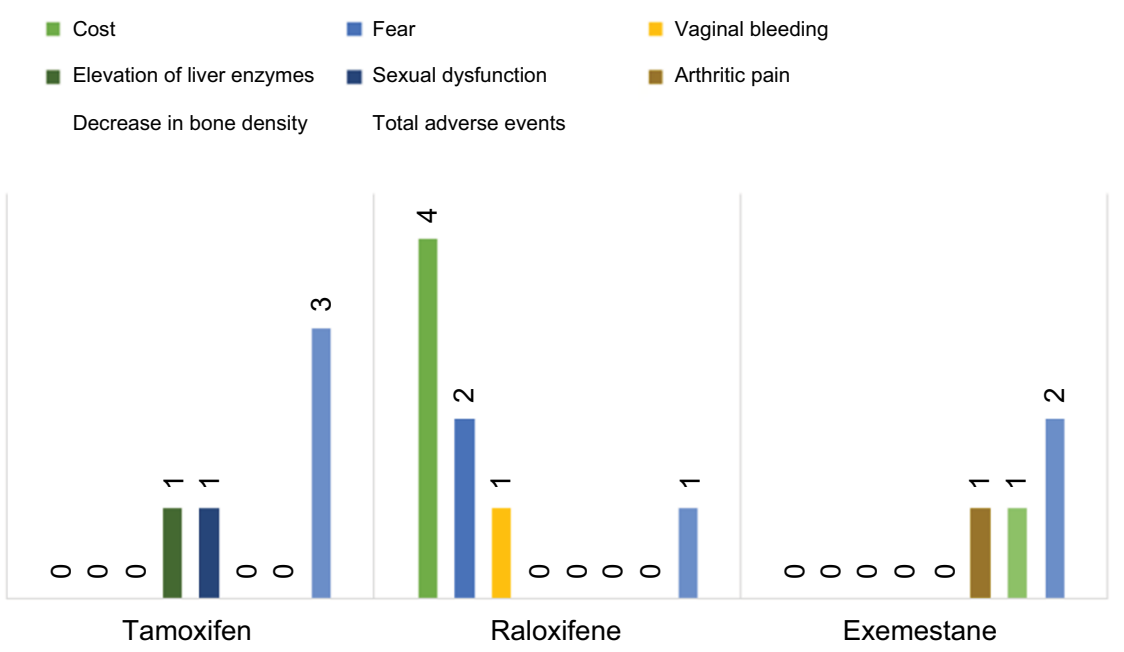

Figure 2 Treatment processes. 


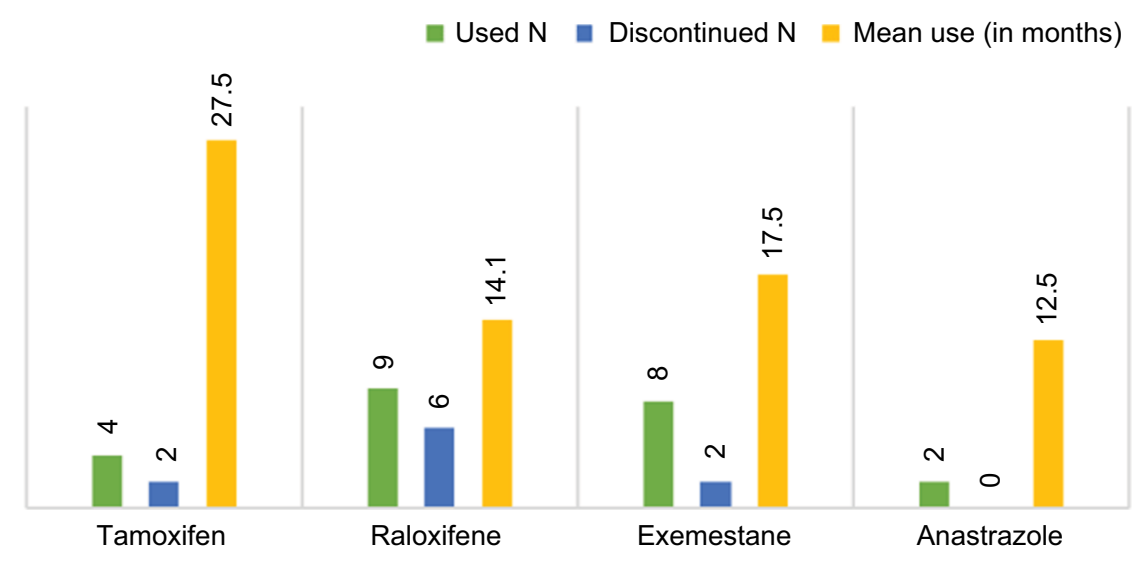

Figure 3 Reasons to discontinue medication.

Table I Clinical characteristics of patients

\begin{tabular}{|l|l|}
\hline Age & $\begin{array}{l}\text { Mean=72.6 years } \\
\text { (range 59-80 years) }\end{array}$ \\
\hline Common comorbid diseases & Number \\
\hline Osteoporosis $(\mathrm{Y} / \mathrm{N})$ & $4 / 19$ \\
\hline HTN $(\mathrm{Y} / \mathrm{N})$ & $19 / 4$ \\
\hline $\mathrm{DM}(\mathrm{Y} / \mathrm{N})$ & $10 / 13$ \\
\hline $\mathrm{CAD}(\mathrm{Y} / \mathrm{N})$ & $3 / 20$ \\
\hline Active smoker $(\mathrm{Y} / \mathrm{N})$ & $0 / 23$ \\
\hline Hysterectomy status $(\mathrm{Y} / \mathrm{N})$ & $14 / 9$ \\
\hline BMI & $\mathrm{M}=32.9 \mathrm{~kg} / \mathrm{m}^{2}$ \\
& $\left(\mathrm{range} 20.6-39 \mathrm{~kg} / \mathrm{m}^{2}\right)$ \\
\hline
\end{tabular}

Abbreviations: BMI, body mass index; CAD, coronary artery disease, DM, diabetes mellitus; HTN, hypertension; $M$, median; N, no; $Y$, yes.

initiation of a chemopreventive agent. Both cancers were estrogen receptor-positive.

\section{Discussion}

Breast cancer chemoprevention in high-risk older women is a missed opportunity to decrease mortality and morbidity from breast cancer. ${ }^{10}$ New research during the last 5 years added AIs to the list of preventive medications in addition to tamoxifen and raloxifene. ${ }^{8,9}$

SERMs such as tamoxifen and raloxifene can increase TEs such as acute deep vein thrombosis, pulmonary embolus, and embolic stroke risk. Also, tamoxifen will increase endometrial cancer risk. AIs such as exemestane and anastrozole may increase the risk for osteoporosis., ${ }^{5,-9}$

An algorithm that aids in the optimal selection of a chemopreventive agent for each woman should decrease the side effect risk and improve acceptance. Despite the small sample size and short duration of the pilot study, the researchers believe the findings to be a meaningful contribution to current knowledge, discussion, and innovation in breast cancer prevention due to the development of algorithm. Concerns about side effects from breast cancer chemoprevention have been associated with lower and inconsistent use of medications. ${ }^{11,19,20}$ For instance, as with other similar studies, the fear of side effects was predictive of discontinuation. Thus, we developed an algorithm logic to minimize side effects, maximize compliance, and extend treatment periods by providing alternatives.

In contrast to many clinical trial settings, real-life circumstances are much more complicated and unpredictable. Our study provided findings from a real-world clinical experience. Except for a few studies that compared tamoxifen to raloxifene, the majority of studies evaluated only one chemopreventive agent. This study used four different agents. To our knowledge, there is no clear published guidance for choosing the optimal medication employing four different agents. Our study proposes an algorithm that guides chemopreventive choice based on potential side effects. Furthermore, the inclusion of four different chemopreventive agents to the algorithm is unique and more comprehensive. In conclusion, primary breast cancer prevention with medications is a proven way to decrease breast cancer risk and can be improved by providing a clear choice process for primary care providers.

\section{Impact statement}

Breast cancer is a major cause of morbidity and mortality in older women. Even with progress in current treatments and screening, breast cancer mortality still remains high in older women. Prevention is a missed opportunity to make an impact. Chemoprevention for breast cancer has drawbacks such as potential side effects from approved medications such as tamoxifen, raloxifene, anastrozole, and exemestane. Careful 
selection of the most appropriate agent will decrease side effects and improve acceptance. The present study provides a novel algorithm for the optimal selection of chemopreventive treatment for older women. Providing this algorithm enhances the choice of the optimal agent for better outcomes, while also improving physician and patient acceptance for prevention. We certify that this work is novel for investigating the optimal choice for an individual older woman by considering multiple drugs according to their side effect profile.

\section{Author contributions}

BZO performed the clinical duties and initial algorithm development and acquired subjects and data. BZO and JBG finalized the algorithm. BZO, JBG, and DLB wrote the paper, analyzed and interpreted the data, and prepared the manuscript. All authors contributed to data analysis, drafting and revising the article, gave final approval of the version to be published, and agree to be accountable for all aspects of the work.

\section{Disclosure}

The authors report no conflicts of interest in this work.

\section{References}

1. Cancer Facts and Figures 2016. American Cancer Society 2016. Cancer. org (online). Available from: http://www.cancer.org/research/cancerfactsstatistics/cancerfactsfigures2016.html. Accessed January 23, 2018.

2. Saenz R. Trends in breast cancer mortality in the United States. PRB (online). Available from: https://www.prb.org/breastcancer/. Accessed October 17, 2018.

3. Estimated Breast Cancer Mortality Worldwide in 2012. Globocan 2012. World Health Organization (Online). Available from: https:/ www.uicc.org/sites/main/files/private/GLOBOCAN2012_Cancer_FactSheets_BreastCancer.pdf. Accessed October 17, 2018.

4. Cancer Facts and Figures 2016. American Cancer Society 2016. Available from: https://www.cancer.org/content/dam/cancer-org/research/ cancer-facts-and-statistics/annual-cancer-facts-and-figures/2016/ cancer-facts-and-figures-2016.pdf. Accessed January 23, 2018.

5. Fisher B, Costantino JP, Wickerham DL, et al. Tamoxifen for the prevention of breast cancer: current status of the National Surgical Adjuvant Breast and Bowel Project P-1 study. J Natl Cancer Inst. 2005;97(22):1652-1662.
6. National Cancer Institute 2011 [webpage on the Internet]. Breast Cancer Risk Assessment Tool [updated May 16, 2011]. Available from: https:// www.cancer.gov/bcrisktool/. Accessed January 23, 2018.

7. Vogel VG, Costantino JP, Wickerham DL, et al; National Surgical Adjuvant Breast and Bowel Project. Update of the national surgical adjuvant breast and bowel project study of tamoxifen and raloxifene (STAR) P-2 trial: preventing breast cancer. Cancer Prev Res (Phila). 2010;3(6): 696-706.

8. Cuzick J, Sestak I, Forbes JF, et al; IBIS-II investigators. Anastrozole for prevention of breast cancer in high-risk postmenopausal women (IBIS-II): an international, double-blind, randomised placebo-controlled trial. Lancet. 2014;383(9922):1041-1048.

9. Goss PE, Ingle JN, Alés-Martínez JE, et al; NCIC CTG MAP.3 Study Investigators.. Exemestane for breast-cancer prevention in postmenopausal women. $N$ Engl J Med. 2011;364(25):2381-2391.

10. Freedman AN, Graubard BI, Rao SR, McCaskill-Stevens W, BallardBarbash R, Gail MH. Estimates of the number of US women who could benefit from tamoxifen for breast cancer chemoprevention. J Natl Cancer Inst. 2003;95(7):526-532.

11. Smith SG, Sestak I, Forster A, et al. Factors affecting uptake and adherence to breast cancer chemoprevention: a systematic review and meta-analysis. Ann Oncol. 2016;27(4):575-590.

12. Wang H, Larson S, Snyder CF, et al. Breast cancer chemoprevention in primary care: Assessing readiness for change. J Clin Oncol. 2016;34(15_suppl):1547.

13. Ropka ME, Keim J, Philbrick JT. Patient decisions about breast cancer chemoprevention: a systematic review and meta-analysis. J Clin Oncol. 2010;28(18):3090-3095.

14. USPTF A and B Recommendations. The Department of Health and Human Services 2015 [updated April 2017]. Available from: https:// www.uspreventiveservicestaskforce.org/Page/Name/uspstf-a-and-brecommendations/. Accessed January 23, 2018.

15. Freedman AN, Yu B, Gail MH, et al. Benefit/risk assessment for breast cancer chemoprevention with raloxifene or tamoxifen for women age 50 years or older. J Clin Oncol. 2011;29(17):2327-2333.

16. Gail MH, Costantino JP, Bryant J, et al. Weighing the risks and benefits of tamoxifen treatment for preventing breast cancer. J Natl Cancer Inst. 1999;91(21):1829-1846.

17. Caprini JA. Thrombosis risk assessment as a guide to quality patient care. Dis Mon. 2005;51(2-3):70-78.

18. Barrett-Connor E, Mosca L, Collins P, et al; Raloxifene Use for The Heart (RUTH) Trial Investigators.. Effects of raloxifene on cardiovascular events and breast cancer in postmenopausal women. $N$ Engl $J$ Med. 2006;355(2):125-137.

19. Taylor R, Taguchi K. Tamoxifen for breast cancer chemoprevention: low uptake by high-risk women after evaluation of a breast lump. Ann Fam Med. 2005;3(3):242-247.

20. Demissie S, Silliman RA, Lash TL. Adjuvant tamoxifen: predictors of use, side effects, and discontinuation in older women. J Clin Oncol. 2001;19(2):322-328.
Breast Cancer - Targets and Therapy

\section{Publish your work in this journal}

Breast Cancer - Targets and Therapy is an international, peerreviewed open access journal focusing on breast cancer research, identification of therapeutic targets and the optimal use of preventative and integrated treatment interventions to achieve improved outcomes, enhanced survival and quality of life for the cancer patient.

\section{Dovepress}

The manuscript management system is completely online and includes a very quick and fair peer-review system, which is all easy to use. Visit http://www.dovepress.com/testimonials.php to read real quotes from published authors. 